\title{
Front Matter: Volume 10068
}

, "Front Matter: Volume 10068," Proc. SPIE 10068, Imaging, Manipulation, and Analysis of Biomolecules, Cells, and Tissues XV, 1006801 (12 April 2017); doi: $10.1117 / 12.2276278$

SPIE. Event: SPIE BiOS, 2017, San Francisco, California, United States 


\title{
Imaging, Manipulation, and Analysis of Biomolecules, Cells, and Tissues XV
}

\author{
Daniel L. Farkas \\ Dan V. Nicolau \\ Robert C. Leif \\ Editors
}

30 January-1 February 2017

San Francisco, California, United States

Sponsored and Published by

SPIE 
The papers in this volume were part of the technical conference cited on the cover and title page. Papers were selected and subject to review by the editors and conference program committee. Some conference presentations may not be available for publication. Additional papers and presentation recordings may be available online in the SPIE Digital Library at SPIEDigitallibrary.org.

The papers reflect the work and thoughts of the authors and are published herein as submitted. The publisher is not responsible for the validity of the information or for any outcomes resulting from reliance thereon.

Please use the following format to cite material from these proceedings:

Author(s), "Title of Paper," in Imaging, Manipulation, and Analysis of Biomolecules, Cells, and Tissues XV, edited by Daniel L. Farkas, Dan V. Nicolau, Robert C. Leif, Proceedings of SPIE Vol. 10068 (SPIE, Bellingham, WA, 2017) Seven-digit Article CID Number.

ISSN: 1605-7422

ISSN: 2410-9045 (electronic)

ISBN: 9781510605770

ISBN: 9781510605787 (electronic)

Published by

SPIE

P.O. Box 10, Bellingham, Washington 98227-0010 USA

Telephone +1 3606763290 (Pacific Time) · Fax +1 3606471445

SPIE.org

Copyright (c) 2017, Society of Photo-Optical Instrumentation Engineers.

Copying of material in this book for internal or personal use, or for the internal or personal use of specific clients, beyond the fair use provisions granted by the U.S. Copyright Law is authorized by SPIE subject to payment of copying fees. The Transactional Reporting Service base fee for this volume is $\$ 18.00$ per article (or portion thereof), which should be paid directly to the Copyright Clearance Center (CCC), 222 Rosewood Drive, Danvers, MA 01923. Payment may also be made electronically through CCC Online at copyright.com. Other copying for republication, resale, advertising or promotion, or any form of systematic or multiple reproduction of any material in this book is prohibited except with permission in writing from the publisher. The CCC fee code is $1605-7422 / 17 / \$ 18.00$.

Printed in the United States of America.

Publication of record for individual papers is online in the SPIE Digital Library.

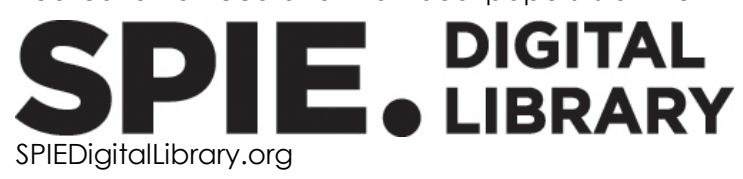

Paper Numbering: Proceedings of SPIE follow an e-First publication model. A unique citation identifier (CID) number is assigned to each article at the time of publication. Utilization of CIDs allows articles to be fully citable as soon as they are published online, and connects the same identifier to all online and print versions of the publication. SPIE uses a seven-digit CID article numbering system structured as follows:

- The first five digits correspond to the SPIE volume number.

- The last two digits indicate publication order within the volume using a Base 36 numbering

system employing both numerals and letters. These two-number sets start with $00,01,02,03,04$, 05, 06, 07, 08, 09, OA, OB ... 0Z, followed by 10-1Z, 20-2Z, etc. The CID Number appears on each page of the manuscript. 


\title{
Contents
}

\author{
vii Authors \\ ix Conference Committee
}

FUNCTIONAL IMAGING I

1006805 Stem cells as anticancer drug carrier to reduce the chemotherapy side effect [10068-4]

10068 0A Quantitative evaluation of blood flow obstruction in microcirculation with sidestream darkfield images [10068-9]

FUNCTIONAL IMAGING II

10068 OC Comparative investigation of stimulus-evoked rod outer segment movement and retinal electrophysiological activity [10068-11]

IMAGE ANALYSIS

10068 ON Novel system for measuring giant spectral images and its application for cancer detection [10068-21]

1006800 3D matching techniques using OCT fingerprint point clouds [10068-22]

$10068 \mathrm{OP}$ Interpreting fiber structure from polarization dependent optical anisotropy [10068-23]

$100680 Q$ Brain vascular image segmentation based on fuzzy local information C-means clustering [10068-24]

10068 OR Use of Gabor filters and deep networks in the segmentation of retinal vessel morphology [10068-25]

10068 OS Comparing methods for analysis of biomedical hyperspectral image data [10068-26]

\section{INSTRUMENTATION}

10068 OY Single-cell analysis of radiotracers' uptake by fluorescence microscopy: direct and droplet approach [10068-32]

$100680 Z$ Dielectrophoretic spectroscopy using a microscopic electrode array [10068-33]

1006811 Femtosecond-laser assisted cell reprogramming [10068-36] 
1006813 Visualization of oxygen transportation in microcirculation by sidestream dark-field oximetry [10068-37]

1006814 Spatial and temporal skin blood volume and saturation estimation using a multispectral snapshot imaging camera [10068-38]

1006815 Component analysis and synthesis of dark circles under the eyes using a spectral image [10068-39]

1006816 Excitation-scanning hyperspectral imaging as a means to discriminate various tissues types [10068-40]

1006818 Evaluation of illumination systems for wide-field hyperspectral imaging in biomedical applications [10068-43]

\section{RARE EVENTS}

10068 1B Optimization of an enhanced ceramic micro-filter for concentrating Escherichia coli in water [10068-45]

HIGH-THROUGHPUT SCREENING

10068 1C Ultrafast, laser-scanning time-stretch microscopy with visible light [10068-46]

POSTER SESSION

10068 IF Quantification of patient-derived 3D cancer spheroids in high content screening images [10068-18]

$100681 \mathrm{H} \quad$ Evaluation of a novel label-free photonic-crystal biosensor imaging system for the detection of prostate cancer cells [10068-54]

$1006811 \quad$ Spheroid imaging of phase-diversity homodyne OCT [10068-55]

$100681 \mathrm{~L}$ Spectroscopic analysis of autofluorescence distribution in digestive organ for unstained metabolism-based tumor detection [10068-58]

10068 IM Laser polarized Xe NMR and MRI at ultra-low magnetic fields [10068-59]

$1006810 \quad$ Usage of CT data in biomechanical research [10068-61]

10068 is Multispectral imaging based on a smartphone with an external C-MOS camera for detection of seborrheic dermatitis on the scalp [10068-65]

$100681 \mathrm{~T}$ In situ temperature control and measurement with femtosecond optical tweezers: offering biomedical application [10068-66] 
$100681 \mathrm{U}$ Efficient femtosecond driven SOX 17 delivery into mouse embryonic stem cells: differentiation study [10068-67]

$100681 \mathrm{~V}$ Testing a high-power LED based light source for hyperspectral imaging microscopy [10068-68]

10068 1W Assignment of vibrational spectral bands of kidney tissue by means of low temperature SERS spectroscopy [10068-69]

$100681 X \quad$ Quantitative phase imaging of platelet: assessment of cell morphology and function [10068-70]

$1006812 \quad$ Referencing techniques for high-speed confocal Fluorescence Lifetime Imaging Microscopy (FLIM) based on Analog Mean-Delay (AMD) method [10068-72]

1006820 Rapid measurement of meat spoilage using fluorescence spectroscopy [10068-73]

1006822 Time-lapse microscopy of lung endothelial cells under hypoxia [10068-75] 
Proc. of SPIE Vol. 10068 1006801-6

Downloaded From: https://www.spiedigitallibrary.org/conference-proceedings-of-spie on 26 Apr 2023 Terms of Use: https://www.spiedigitallibrary.org/terms-of-use 


\section{Authors}

Numbers in the index correspond to the last two digits of the seven-digit citation identifier (CID) article numbering system used in Proceedings of SPIE. The first five digits reflect the volume number. Base 36 numbering is employed for the last two digits and indicates the order of articles within the volume. Numbers start with 00, 01, 02, 03, 04, 05, 06, 07, 08, 09, 0A, OB...0Z, followed by 10-1Z, 20-2Z, etc.

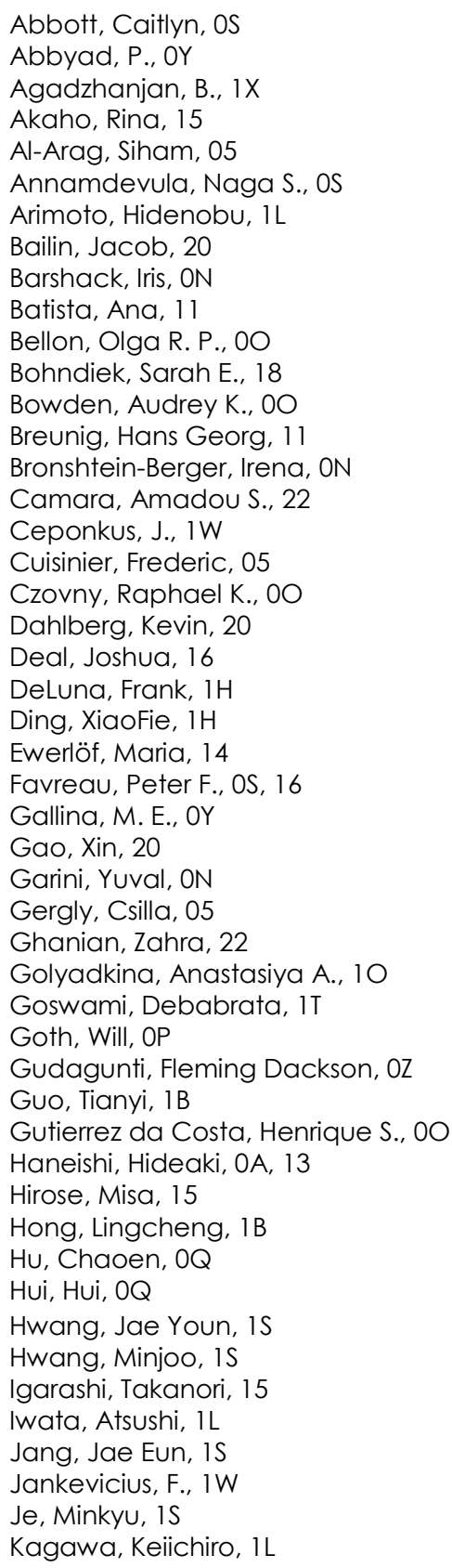

Kang, Mi-Sun, $1 \mathrm{~F}$

Kawahira, Hiroshi, 13

Kawahito, Shoji, 1L

Kim, Byungyeon, 1 Z

Kim, Jihun, 1S

Kim, Manjae, is

Kim, Myoung-Hee, IF

Kim, Sewoong, $1 \mathrm{~S}$

Kim, T. J., OY

Kirillova, Irina $V_{\text {., }} 10$

Kirmani, Syed Abdul Mannan, OZ

Klomkaew, Phiwat, $1 \mathrm{~V}$

Kondouri, Ganesh, 22

König, Karsten, 11

Kossovich, Leonid Y., 10

Kumagai, Hiroshi, $1 \mathrm{M}$

Kumar, Pramod, ON

Kurata, Tomohiro, 0A, 13

Lakshminarayanan, Vasudevan, OR

Lall, Malvika, 16

Larsson, Marcus, 14

Leavesley, Silas J., OS, 16, 1V

Lee, Dong Hun, is

Lee, Minsuk, $1 \mathrm{Z}$

Lee, Seungrag, 12

Lemboumba, Satuurnin Ombinda, IU

Leopold, Henry A., OR

Liang, Xiao, $0 Q$

Lima, Ivan T., Jr., OZ

Linder, Moshe, ON

Liv, Xia, $O Q$

Lopez, Carmen, 16

LU, Yiming, OC

Luthman, A. Siri, 18

Lyfenko, R., $1 \mathrm{X}$

Maaza, Malik, $1 \mathrm{U}$

Manoto, Sello Lebohang, $1 U$

Marin, Irina, ON

Mayer, Arnaldo, ON

Mayes, Sam A., IV

Mehrvar, Shima, 22

Metelin, Vladislav, $1 \mathrm{X}$

Middendrop, Elodie, 05

Mondal, Dipankar, $1 T$

Mthunzi-Kufa, Patience, $1 \mathrm{U}$

Nawarathna, Dharmakeerthi, $\mathrm{OZ}$

Necula, Daniela, ON

Oda, Shigeto, 13

Ohnishi, Takashi, 0A, 13 
Ojima, Nobutoshi, 15

Orchard, Jeff, OR

Osawa, Kentaro, 11

Park, Byungjun, $1 Z$

Pratx, G., OY

Pucetaite, M., IW

Radzvilaite, M., IW

Ranji, Mahsa, 22

Rhee, Seon-Min, IF

Rich, Thomas C., OS, 16, $1 \mathrm{~V}$

Sablinskas, V., IW

Sacks, Michael S., OP

Safonov, Roman A., 10

Salehi, Hamideh, 05

Salerud, E. Göran, 14

Sanomura, Yoji, 1L

Sawyer, Travis W., 18

Senda, Naoko, 11

Seo, Ji-Hyun, $1 \mathrm{~F}$

Shotan, Zav, ON

Silva, Luciano, 00

Smith, Jason, 20

Steiner, G., IW

Sun, Lu-Zhe, $1 \mathrm{H}$

Sweat, Brenner, OS

Takahashi, Minori, OA, 13

Takeda, Shun, IM

Tanaka, Shinji, $1 \mathrm{~L}$

Thobakgale, Lebogang, IU

Tian, Jie, OQ

Tsia, Kevin K., 1C

Tsumura, Norimichi, 15

Tuerkcan, S., OY

Tunnell, James W., OP

Uchugonova, Aisada, 11

Urboniene, $\mathrm{V} ., \mathrm{IW}$

Vasilenko, Irina, $1 \mathrm{X}$

Vasquez, J., OY

Velicka, M., IW

Velmanickam, Logeeshan, $0 Z$

Vlasova, Elizaveta, 1X

Wang, Benquan, $0 \mathrm{C}$

Weber, David S., 16

Won, Youngjae, $1 \mathrm{Z}$

Wong, Kenneth K. Y., 1C

Wu, Binlin, 20

Wu, Jianglai, $1 C$

$X U$, Changaing, 1B

Yan, Wenwei, 1C

Yang, Xin, $O Q$

Yao, Xincheng, OC

Ye, Jing Yong, $1 \mathrm{H}$

Yoshida, Shigeto, $1 \mathrm{~L}$

Zelek, John, OR

Zhang, Yushan, 1B

Zimmerman-Moreno, Gali, ON 


\title{
Conference Committee
}

\author{
Symposium Chairs
}

James G. Fujimoto, Massachusetts Institute of Technology

(United States)

R. Rox Anderson, Wellman Center for Photomedicine, Massachusetts General Hospital (United States) and Harvard Medical School

(United States)

Program Track Chairs

Ammasi Periasamy, University of Virginia (United States)

Daniel L. Farkas, University of Southern California (United States) and SMI (United States)

Conference Chairs

Daniel L. Farkas, University of Southern California (United States) and SMI (United States)

Dan V. Nicolau, McGill University (Canada)

Robert C. Leif, Newport Instruments (United States)

\section{Conference Co-chairs}

James F. Leary, Purdue University (United States)

Attila Tarnok, Universität Leipzig (Germany)

\section{Conference Program Committee}

Vadim Backman, Northwestern University (United States)

Christopher H. Contag, Stanford University School of Medicine (United States)

Paul M. W. French, Imperial College London (United Kingdom)

Yuval Garini, Bar-Ilan University (Israel)

Jae Youn Hwang, DGIST (Korea, Republic of)

Charles P. Lin, Wellman Center for Photomedicine (United States)

Sacha Loiseau, Mauna Kea Technologies (France)

Ramesh Raghavachari, U.S. Food and Drug Administration

(United States)

Sebastian Wachsmann-Hogiu, University of California, Davis

(United States)

Warren S. Warren, Duke University (United States) 
Session Chairs

1 Cytomics and Histomics

Daniel L. Farkas, University of Southern California (United States) and SMI (United States)

2 Functional Imaging I

Daniel L. Farkas, University of Southern California (United States) and SMI (United States)

3 Functional Imaging II

Attila Tárnok, Universität Leipzig (Germany)

$4 \quad$ Functional Imaging Organoids

Attila Tárnok, Universität Leipzig (Germany)

5 Regenerative Medicine

Fartash Vasefi, SMI (United States)

Robert C. Leif, Newport Instruments (United States)

6 Image Analysis

Attila Tárnok, Universität Leipzig (Germany)

7 Biomedical Imaging using a DMD or other Light Structuring Devices: Joint Session with Conferences 10068 and 10117

Michael R. Douglass, Texas Instruments Inc. (United States)

8 Instrumentation

Robert C. Leif, Newport Instruments (United States)

9 Multispectral Imaging

Dan V. Nicolau, McGill University (Canada)

10 Rare Events

Dan V. Nicolau, McGill University (Canada)

11 High-throughput Screening

Dan V. Nicolau, McGill University (Canada) 